\title{
El sector de Cerámica y Vidrio en la Región de Murcia y España durante el siglo XIX
}

\author{
J. M. MARTÍNEZ CARRIÓN \\ Departamento de Economía Aplicada. Facultad de Economía y Empresa. \\ Universidad de Murcia Campus de Espinardo. 30100 Espinardo (Murcia)
}

\begin{abstract}
El trabajo destaca la relevancia histórica que adquirió el sector que tradicionalmente engloba las industrias de 'cerámica, vidrio y materiales de construcción' en la región de Murcia y su contribución a la industrialización española del siglo XIX. Primeramente, se examina la aportación del sector a la estructura fabril de la Región de Murcia y a la industria española. A partir de fuentes fiscales se muestra la especialización industrial que el sector adquirió en la segunda mitad del siglo XIX, que comparte posiciones similares junto con las industrias metalúrgicas y mineras, muy por encima de los sectores alimentarios y textiles. Asimismo, se analizan los factores de localización industrial, la distribución espacial de la producción y los cambios producidos en sus diferentes ramas entre 1856 y 1905, con el fin de valorar los aspectos más dinámicos y su influencia en la geografía española. Concluye señalando el protagonismo del sector durante el periodo de la Restauración.
\end{abstract}

Palabras Clave: Industria cerámica y vidrio, Revolución Industrial, España, Región de Murcia, Siglo XIX.

\section{The sector of Ceramics and Glass in the Region of Murcia and Spain during the XIXth century}

This investigation emphasizes the historic relevance the sector traditionally comprising ceramics, glass, and building industry played in the Region of Murcia as well as its contribution to Spanish industrialization within the nineteenth century. First of all, it is analyzed the contribution of this sector to the manufacturing framework in both the Region of Murcia and the Spanish industry. From official records it is shown the industrial expecialization this sector reached during the second half of the XIXth century, sharing positions with mining and metallurgist and quite over food and textile sectors. Meanwhile, factors of industrial localization, spatial distribution of production, and changes occurred in the different sectors between 1856 and 1905 to evaluate the more dynamic aspects and their effects on the Spanish geography are analyzed. We conclude emphasizing the importance of this sector during the Restoration.

Key words: Ceramics and Glass Industry, Industrial Revolution, Spain, Region of Murcia, XIXth Century.

\section{INTRODUCCIÓN}

El proceso de industrialización en España arranca en la primera mitad del siglo XIX, aunque algunos sectores se remontan a fines del siglo XVIII. La industria fabril algodonera en Cataluña es la principal muestra del arraigo temprano de la industrialización en la península y, junto a la industria del hierro en el País Vasco, se erigieron en los símbolos de la Revolución Industrial en España. Fenómeno que fue simultáneo a la mayoría de los países que se industrializaron en el curso del largo siglo XIX ${ }^{1}$. Sin embargo, en la segunda mitad de dicho siglo, la mecanización se difundió en casi todos los sectores, ya provinieran de las industrias de bienes de consumo o de las industrias de bienes de producción. La industrialización alcanzó a los más tradicionales, desencadenado con ello cambios en los modelos de gestión, comercialización y organización del trabajo. El sector que considera a las industrias de 'cerámica, vidrio y materiales de construcción' no escapó a dicho fenómeno, aunque presentó aspectos evolutivos muy desiguales.

El ascenso de la burguesía y los cambios institucionales, de un lado; el incremento de la renta y los cambios tecnológicos, de otro $y$, en fin, un conjunto de cambios sociales, económicos y políticos hicieron posible que la industrialización se difundiera en la producción de materiales de cerámica y vidrio, sec- tores hasta entonces muy artesanales. Al amparo del crecimiento demográfico, la urbanización, la ampliación de los mercados y los cambios producidos en los estilos de vida, la oferta de productos cerámicos y de artículos de cristal y vidrio se diversificó. La demanda de consumo de bebidas y las necesidades de la industria química-farmacéutica provocaron un enorme tirón en la fabricación de botellas y, con otros envases, presionó sobre la oferta de vidrios huecos. La demanda de cristales para ventanas, de espejos para equipos equipamientos domésticos y la expansión de la industria de la construcción hicieron lo propio con la de vidrios planos. El número de fábricas se multiplicó $\mathrm{y}$, a menudo, conformaron pequeños complejos cerámico-vidrieros que ahora, probablemente, se asemejarían a los distritos industriales. Con ellos, el tejido industrial se diversificó y ensanchó y, lo que es más importante, se fortaleció en regiones de escasa tradición fabril. Desde la década de 1840, las cerámicas y los vidrios conocieron un verdadero impulso industrial. Entre 1870 y la Primera Guerra Mundial, el sector entra en un proceso de reestructuración y redistribución geográfica, coincidiendo con la difusión de tecnologías propias de los primeros estadios de la industrialización y punteras de la Segunda Revolución Industrial. 
Siendo un sector tradicional y muy modesto, emplazado frecuentemente en torno a las fuentes de aprovisionamiento de materias primas y energía, las industrias de productos cerámicos y vítreos experimentaron una auténtica eclosión en las décadas centrales del siglo XIX y percibieron un proceso de transformaciones técnicas y económicas durante la etapa de la Restauración. A las puertas del siglo XX, la reestructuración afectó con más intensidad al sector de las industrias del vidrio, que afronta una coyuntura marcada por un proceso de internacionalización y tendencia al oligopolio para hacer frente a la competencia. Como en otros países, en España se manifiesta bajo los signos de la concentración empresarial y la cartelización liderados, en este caso, por el grupo francés de Saint-Gobain.

\section{OBJETIVOS. MÉTODOS Y FUENTES.}

Este trabajo tiene como objetivo profundizar en el conocimiento del proceso histórico de la industrialización del sector de la cerámica y el vidrio en España y destacar, asimismo, la aportación murciana a la industria española de la segunda mitad del siglo XIX. Siendo relevante en la historia económica española y, sobre todo, de algunas regiones, el sector ha recibido un escaso tratamiento y a menudo ha caído en el olvido por parte de los historiadores económicos, a diferencia del trato acogido por los historiadores del arte. Un estudio ponderado de la industrialización regional refleja el papel que desempeñó el sector que, genéricamente, se denominó como "cerámica, vidrio, yeso y cal". Éste engloba la fabricación de porcelana, loza, cristal, vidrio y otros productos cerámicos, así como materiales de construcción, yeso y cal. El espectro de actividades fabriles, como puede deducirse, es demasiado amplio y cubre una oferta de productos que van desde los más exquisitos y refinados objetos de decoración y ornamentación de cerámica y vidrio, usados en los hogares de la burguesía y de las familias adineradas, hasta los materiales de construcción más comunes utilizados en las viviendas del proletariado y el campesinado.

En este trabajo se presenta un balance general de la industrialización regional del sector a través de fuentes fiscales que arrojan información muy valiosa sobre la estructura fabril en la España de la segunda mitad del siglo XIX. Los resultados generales obtenidos para España son comparados con los de la Región de Murcia y revelan la importancia relativa de los sectores fabriles y los progresos de la especialización productiva. El objetivo es señalar su importancia fabril en la región de Murcia y situarla en el contexto español del Ochocientos. Las Estadísticas de Contribución Industrial y de Comercio (en adelante, ECI) de 1856 a $1905^{2}$ sirven de base para ponderar, aunque sea grosso modo, la participación de cada sector en el total fabril y permiten conocer de manera muy fiable los cambios producidos en la distribución territorial del sector en España a lo largo de dicho periodo, con la excepción de las provincias vascas y Navarra cuyos datos no se contemplan en dichas estadísticas dadas las peculiaridades de exención fiscal de dichas regiones históricas.

Siendo datos fiscales que nacieron a raíz de la modernización del sistema impositivo propuesto por Mon y Santillán, en 1845 , se presupone que hubo alguna ocultación de la riqueza industrial. Esto podría introducir un sesgo en los resultados del estudio. Sin embargo, se ha señalado que la ocultación debió afectar en proporciones similares a todos los sectores fabriles y aparatos o artefactos mecánicos instalados en ellos ${ }^{3}$.
De ahí que sean de enorme utilidad para los historiadores de la industrialización española. Ante la ausencia de información estadística sobre el valor añadido o el producto generado por cada sector, como ocurre con las estadísticas industriales en la actualidad, las fuentes fiscales que aquí se manejan constituyen, por ahora, el único procedimiento para averiguar la evolución histórica ponderada de las ramas industriales en un período crucial de la industrialización española, como es el de la segunda mitad del Ochocientos.

Los resultados obtenidos son consistentes con la información cuantitativa y cualitativa de numerosos trabajos arrojados por la literatura especializada ${ }^{4}$. En contra de lo sostenido en la historiografía ${ }^{5}$, el trabajo destaca el peso de las industrias que tributaban por el sector de la cerámica y vidrio en la Región de Murcia. Se pone de relieve que en dicha región se dibujaron dos grandes líneas de especialización industrial en el curso del último tercio del siglo XIX; una, provenía del boom minero y fomentó el desarrollo de las industrias de fundición y las metalúrgicas (plomo y hierro, principalmente y por este orden); la otra, no menos importante, pero casi desconocida, se asentaba en la cerámica y el vidrio. Rescatar, por tanto, el papel que las últimas desempeñaron en la historia industrial es el objetivo principal del trabajo y cuyos datos son expuestos a continuación, en el capítulo 3, señalándose la aportación del ramo en su conjunto tanto en la industria murciana como la industrialización española. En el mismo apartado se analizan los índices de especialización industrial y la contribución de las industrias murcianas al sector en España. El siguiente apartado enfatiza los factores de localización industrial y los cambios producidos en la distribución regional; con ello se pretende señalar los determinantes del sector en los inicios de la industrialización española y su implantación territorial entre 1856 y 1905, fechas decisivas para su definitiva emplazamiento. Por último, se analiza la composición interna del sector en España, aportando evidencias acerca de los cambios que se produjeron como consecuencia de las transformaciones urbanas, económicas y sociales. Se apunta el declive relativo de las industrias más tradicionales de la construcción, como tejas, ladrillos, tinajas y vasijas convencional, y el alza de las nuevas ramas: azulejería, losetas hidráulicas, piedra, cristal y vidrio.

\section{EL SECTOR DE LA CERÁMICA Y EL VIDRIO EN LA REGION DE MURCIA Y SU APORTACIÓN A LA INDUSTRIA ESPAÑOLA DEL SIGLO XIX}

Como se ha señalado, la historia industrial de la región murciana ha estado durante mucho tiempo ligada a la minería y la metalurgia del plomo. Junto a Andalucía, Murcia y, en particular, las sierras de Cartagena y La Unión, Mazarrón y Águilas, cobraron un fuerte protagonismo durante la segunda mitad del siglo XIX al amparo de las intensas extracciones de minerales de plomo, primero, y férricos ${ }^{6}$. La atención prestada en la última década a la reconstrucción de una historia industrial regional más ponderada ha revelado, sin embargo, la importancia de sectores y subsectores a menudo poco conocidos ${ }^{7}$. Partiendo de niveles muy modestos, el de la "cerámica, vidrio y construcción" llega a sostener un fuerte desarrollo en el tejido fabril de la Región de Murcia y en el de otras regiones. Aunque la producción de alimentos y tejidos constituía la principal actividad de las manufacturas, como en casi toda la geografía española hasta mediados del siglo XIX, los datos revelan que las industrias de cerámica, vidrio y materiales de construcción alcanzaron un peso significativo, mayor incluso que el del sec- 
TABLA 1. DistribuCión SECTORIAL DE LA INDUSTRIA EN MURCIA (1) Y ESPAÑA (2), 1856-1905

\begin{tabular}{|c|c|c|c|c|c|c|c|c|c|c|c|c|}
\hline Sectores & \multicolumn{2}{|c|}{1856} & \multicolumn{2}{|c|}{1863} & \multicolumn{2}{|c|}{1890} & \multicolumn{2}{|c|}{1895} & \multicolumn{2}{|c|}{1900} & \multicolumn{2}{|c|}{1905} \\
\hline & (1) & (2) & (1) & (2) & (1) & (2) & (1) & (2) & (1) & (2) & (1) & (2) \\
\hline Alimenticias & 64. & 55.7 & 57.65 & 52.8 & 54.14 & 43.6 & 43.5 & 40.2 & 41.6 & 40.3 & 32.4 & 38.97 \\
\hline Textiles & 7.5 & 23.6 & 9.62 & 23.0 & 8.12 & 23.7 & 9.1 & 23.6 & 10.8 & 26.6 & 10.8 & 26.6 \\
\hline Metalúrgicas & 12. & 3.2 & 14.3 & 5.0 & 14.3 & 6.6 & 17.4 & 7.1 & 18.9 & 8.1 & 26.8 & 9.22 \\
\hline Químicas & 4.9 & 3.5 & 3.7 & 4.2 & 4.4 & 6.3 & 6.6 & 5.9 & 4.4 & 5.7 & 4.4 & 5.9 \\
\hline Papel-Gráficas & 1.5 & 2.3 & 1.1 & 2.5 & 2.9 & 4.0 & 4.4 & 5.3 & 5.2 & 5.0 & 6.1 & 4.9 \\
\hline Cerámica, vidrio, yeso y cal & 7.6 & 5.3 & 10.9 & 4.1 & 11.7 & 4.8 & 12.1 & 4.2 & 11.1 & 4.0 & 10.9 & 4.0 \\
\hline Madera & -- & 1.2 & -- & 1.1 & 1.5 & 2.5 & 2.9 & 2.5 & 2.5 & 3.2 & 4.5 & 4.0 \\
\hline Cuero-Calzado & 1.1 & 3.8 & 1.8 & 4.0 & 0.6 & 3.2 & 2.2 & 3.1 & 4.3 & 2.9 & 1.4 & 2.8 \\
\hline Diversas & 0.4 & 1.1 & 1.0 & 2.9 & 2.3 & 4.9 & 1.7 & 5.0 & 1.4 & 4.1 & 2.6 & 3.2 \\
\hline TOTAL & 100 & 100 & 100 & 100 & 100 & 100 & 100 & 100 & 100 & 100 & 100 & 100 \\
\hline \multicolumn{13}{|c|}{ Columnas (1) Murcia, (2) Eşaña. } \\
\hline $\begin{array}{l}\text { Fuente: Elaboración propia a } \\
\text { Comercio (en adelante, ECI), }\end{array}$ & & $\mathrm{la}(\mathrm{s})$ & Estadís & & & & & & & & & \\
\hline
\end{tabular}

TABLA 2. CONTRIBUCIÓN MURCIANA A LA INDUSTRIA ESPAÑOLA (\%/TOTAL) ENTRE 1856 Y 1905.

\begin{tabular}{|lcccccc|}
\hline Sectores & $\mathbf{1 8 5 6}$ & $\mathbf{1 8 6 3}$ & $\mathbf{1 8 9 0}$ & $\mathbf{1 8 9 5}$ & $\mathbf{1 9 0 0}$ & $\mathbf{1 9 0 5}$ \\
\hline Alimentarias & 2.00 & 1.70 & 2.54 & 1.67 & 1.57 & 0.76 \\
Textiles & 0.53 & 0.65 & 0.70 & 0.54 & 0.61 & 0.37 \\
Metalúrgicas & 6.39 & 4.45 & 4.40 & 3.75 & 3.73 & 2.65 \\
Químicas & 2.63 & 1.36 & 1.40 & 1.72 & 1.20 & 0.67 \\
Papel-Gráficas & 1.40 & 0.71 & 1.45 & 1.27 & 1.52 & 1.12 \\
Cerámica, vidrio y cal & 2.43 & 4.15 & 4.94 & 4.39 & 4.16 & 2.49 \\
Madera & -- & -- & 1.23 & 1.74 & 1.21 & 1.01 \\
Cuero-Calzado & 0.49 & 0.70 & 0.39 & 1.11 & 2.24 & 0.45 \\
Diversas & 0.19 & 0.50 & 0.96 & 0.54 & 0.36 & 0.74 \\
(\%) DE MURCIA / ESPAÑA & $\mathbf{1 . 7 0}$ & $\mathbf{1 . 5 1}$ & $\mathbf{2 . 0 0}$ & $\mathbf{1 . 5 4}$ & $\mathbf{1 . 5 1}$ & $\mathbf{0 . 9 1}$ \\
\hline
\end{tabular}

FUENTE: ELABORACIÓN PROPIA A PARTIR DE LAS ECI.

TABLA 3. INDICES DE ESPECIALIZACIÓN DE LA INDUSTRIA DE LA REGIÓN MURCIANA, 1857-1905 (BASE 100 = ESPAÑA)

\begin{tabular}{|lrrrrrr|}
\hline Sectores & $\mathbf{1 8 5 6}$ & $\mathbf{1 8 6 3}$ & $\mathbf{1 8 9 0}$ & $\mathbf{1 8 9 5}$ & $\mathbf{1 9 0 0}$ & $\mathbf{1 9 0 5}$ \\
\hline Alimenticias & 116.1 & 109.1 & 124.1 & 108.2 & 103.2 & 83.3 \\
Textil & 31.8 & 41.7 & 34.2 & 38.5 & 40.6 & 40.7 \\
Metalurgia & 381.2 & 286.0 & 216.6 & 245.0 & 233.3 & 291.3 \\
Química & 140.0 & 88.1 & 69.8 & 111.8 & 77.2 & 74.6 \\
Papel-Gráficas & 65.2 & 44.0 & 72.5 & 83.0 & 104.0 & 124.5 \\
Cerámica- vidrio & $\mathbf{1 4 3 . 4}$ & $\mathbf{2 6 5 . 8}$ & $\mathbf{2 4 3 . 7}$ & $\mathbf{2 8 8 . 0}$ & $\mathbf{2 7 7 . 5}$ & $\mathbf{2 7 2 . 5}$ \\
Madera & - & - & 60.0 & 116.0 & 78.1 & 112.5 \\
Curtido-Calzado & 28.9 & 45.0 & 18.7 & 70.9 & 148.3 & 50.0 \\
Diversas & 36.3 & 34.5 & 46.9 & 34.0 & 34.1 & 81.2 \\
\hline
\end{tabular}

Fuente: Elaboración propia a partir de las ECI

tor de la madera y el calzado a la altura de 1900, y aún por encima de las químicas y de las industrias papelera y de artes gráficas hasta los comienzos de la Restauración, ver Tabla 1.

La industria fabril murciana a mediados del siglo XIX se asemeja, por su composición y rasgos tecnológicos, a la de otras regiones agrarias españolas en donde el peso de las alimentarias supone dos tercios del conjunto de las utilidades. La única excepción a este tradicional panorama lo constituye el auge de las industrias metalúrgicas ligadas al boom de la minería. Pero, en tercer lugar, y por encima de los "textiles" -sector emblemático por el peso histórico de la industria sedera-, se sitúan las ramas fabriles de "cerámica, vidrio, yeso y cal". A lo largo del último tercio del siglo XIX se asiste a un fuerte declive de las posiciones relativas del sector alimentario, cuyo origen reside no sólo en la reestructuración productiva del sector, durante y tras la denominada 'crisis agraria finisecular' (1875-1895), sino al empuje de las "metalúrgicas" y las "cerámicas", cuya posición relativa aumenta en la década de 1890 y a comienzos del siglo XX. Desde mediados de la centuria pasada ambos sectores registran notorios avances, mantienen su posición a comienzos del siglo XX y alcanzan porcentajes muy superiores a los que dichos sectores consiguen en el conjunto de la industria española.

La importancia del sector que se analiza aumenta cuando se comprueba la aportación murciana, medida en términos relativos, a los diferentes sectores fabriles de España entre 1856 y 1905. Los datos de la tabla 2 confirman el papel que desempeñan las fábricas de fundición y metalúrgicas en la industria nacional hasta la década de 1880; pero también el protagonismo relativo del sector de las cerámicas, vidrio y materiales de construcción que duplican su participación muy por encima de la cuota porcentual regional. Contribuyen a ella, en las décadas finales del siglo XIX, más de un centenar de pequeñas fábricas de tinajas y vasijería vidriada, tejas, ladrillos y baldosas, losetas hidráulicas, de yeso o cal, pero sobresale, por encima de todas, la Fábrica de Cristal de Santa Lucía, que figura, ya en 1895, entre las primeras de su especialidad. Los datos globales revelan la importancia de los negocios en el sector durante la etapa finisecular.

De acuerdo con las cuotas de contribución y no por contribuyentes, Murcia pasa del decimoquinto puesto en el ranking provincial, en 1856, a escalar el quinto lugar durante la década de 1890, detrás de Barcelona, Valencia, Sevilla y Madrid. El hecho merece la pena ser destacado, pues revela los progresos alcanzados en apenas unas décadas por una provincia sin tradición histórica en el sector, a diferencia de las señaladas arriba, que albergaban los principales centros urbanos y de consumo del país. A ellas habría que añadir Bilbao que las estadísticas fiscales no contemplan por razones antes señaladas. En cualquiera de los casos, fuera quinta o sexta posición, el hecho es que Murcia se encuentra entre las grandes provincias con mayor contribución económica al sector.

La contribución murciana a las industrias españolas del sector de la cerámica y el vidrio y la especialización fabril queda patente en las Tablas 2 y 3 . Este último procedimiento, aunque algo tosco de acuerdo con la metodología seguida -se trata de una sencilla operación de cociente entre la parte alícuota murciana y la española- nos permite seguir la pista de la pujanza del sector en el periodo de la primera Revolución Industrial en España. Los datos son contundentes. Murcia es una región que se industrializa tempranamente de la mano de la minería, asentándose en ella, desde 1840, fábricas de fundición de plomo y metalúrgicas, que alcanzan su mayor apogeo en la década de 1850-60, coincidiendo con el desarrollo de las industrias mineras asentadas en la Sierra de Cartagena y, añadiéndose a finales del siglo XIX, las de Mazarrón. Pero la metalurgia estuvo acompañada por la expansión del sector de la cerámica y el vidrio que, aprovechando las economías de escala y las ventajas comparativas que proporcionaba la eclosión minera (energía, materias primas, rentas de situación, técnicos, capitales, mano de obra) tuvo su mayor esplendor en el complejo vidriero-cerámico de Cartagena. 


\section{FACTORES DE LOCALIZACIÓN Y CAMBIOS EN LA DISTRIBUCIÓN REGIONAL DEL SECTOR}

De una u otra forma, en las décadas centrales del siglo XIX, las industrias "cerámicas y vítreas" estaban presentes en la España meridional y del interior, pero acabaron imponiéndose en la España mediterránea a comienzos del siglo XX (Figuras 1, 2 y 3; Tabla 4). A lo largo de la segunda mitad del siglo XIX, Asturias fue la única región que mostró un claro empuje del sector en un contexto generalizado de atonía y abandono relativo de las posiciones alcanzadas en la década de 1850 en la España septentrional y del interior. Ni siquiera Andalucía con su fuerte tradición y especialización ceramista pudo resistir el embate de la competitividad a finales del siglo XIX.

En 1856, el sector ocupaba, por orden de importancia, el tercer y cuarto lugar en la mayoría de las regiones españolas. El número de contribuyentes por todas las modalidades fabriles del sector fue de 1.646, con una cuota global de 518.279 reales, equivalente a 129.570 pesetas $^{8}$. En 1900, la cuota española por fabricación en los diversos ramos era de 282.033 pesetas, registrándose los avances más significativos en Asturias, Canarias, Cantabria y Murcia, de acuerdo con los datos de la columna 7 de la Tabla 4. Pero por su peso fiscal, la primera y la última son las que más descuellan en su crecimiento. No en vano, entre ambos periodos, emerge con fuerza la industria vidriera -Asturias ocupa la segunda posición industrial en concepto de vidrio, tras la provincia de Barcelona-, y se expanden las fábricas de "loza entrefina" y de "objetos refractarios" -Asturias asciende, también, en estos ramos al primer puesto-.

Los datos refuerzan la especialización que el sector en su conjunto muestra en las regiones mediterráneas. La diversificación del tejido industrial y la especialización de la "cerámica y el vidrio" son caras de la misma moneda. Cataluña y el País Valenciano aumentan su participación; la segunda región, junto con Asturias y Murcia cobran un fuerte empuje en la década de 1880, mientras las tres primeras prosiguen su marcha ascendente, incluso, en la primera década del siglo $X X$. La región de Murcia comienza su repliegue en la década de

TAbla 4. Distribución Regional del Sector de CerámicA, Vidrio en ESPAÑA, 1856-1905.

\begin{tabular}{|l|r|r|r|r|r|r|r|}
\hline \multicolumn{1}{|c}{$\begin{array}{c}\text { Comunidades } \\
\text { Autónomas }\end{array}$} & \multicolumn{1}{c|}{$\begin{array}{c}\mathbf{1 8 5 6} \\
\mathbf{( 1 )}\end{array}$} & \multicolumn{1}{c|}{$\begin{array}{c}\mathbf{1 8 7 9} \\
\mathbf{( 2 )}\end{array}$} & \multicolumn{1}{c|}{$\begin{array}{c}\mathbf{1 8 9 0} \\
\mathbf{( 3 )}\end{array}$} & \multicolumn{1}{c|}{$\begin{array}{c}\mathbf{1 8 9 5} \\
\mathbf{( 4 )}\end{array}$} & \multicolumn{1}{c|}{$\begin{array}{c}\mathbf{1 9 0 0} \\
\mathbf{( 5 )}\end{array}$} & \multicolumn{1}{c|}{$\begin{array}{c}\mathbf{1 9 0 5} \\
\mathbf{( 6 )}\end{array}$} & \multicolumn{1}{c}{$\begin{array}{c}\text { \% (1)/(5) } \\
\mathbf{( 7 )}\end{array}$} \\
\hline Andalucía & 24.58 & 23.92 & 24.99 & 21.97 & 22.43 & 18.32 & -8.74 \\
Aragón & 5.02 & 5.05 & 4.87 & 3.82 & 3.33 & 4.29 & -33.66 \\
Asturias & 0.55 & 3.03 & 2.65 & 3.92 & 4.25 & 5.94 & 672.72 \\
Baleares & 2.25 & 2.75 & 1.46 & 1.80 & 1.43 & 1.21 & -36.44 \\
Canarias & 0.18 & 0.32 & 0.22 & 0.42 & 0.47 & 0.48 & 161.11 \\
Cantabria & 0.59 & 0.96 & 1.26 & 2.20 & 1.26 & 1.77 & 113.55 \\
Castilla-Mancha & 10.30 & 7.97 & 6.17 & 4.85 & 5.38 & 6.33 & -47.76 \\
Castilla-León & 12.44 & 11.79 & 9.78 & 9.47 & 8.60 & 7.75 & -30.86 \\
Cataluña & 15.71 & 15.52 & 15.86 & 21.62 & 24.91 & 25.46 & 58.58 \\
Extremadura & 3.80 & 6.92 & 3.51 & 3.21 & 2.89 & 2.63 & -23.94 \\
Galicia & 2.99 & 2.78 & 1.22 & 0.96 & 0.85 & 0.82 & -71.57 \\
Madrid & 8.83 & 4.92 & 7.60 & 6.76 & 6.84 & 8.54 & -22.53 \\
Murcia & 2.43 & 1.91 & 4.86 & 4.39 & 4.16 & 2.49 & 71.19 \\
Pá́s Valenciano & 9.79 & 11.28 & 14.66 & 13.72 & 12.36 & 13.26 & 26.25 \\
Rioja & 0.51 & 0.82 & 0.84 & 0.83 & 0.83 & 0.64 & 62.74 \\
TOTAL & 100 & 100 & 100 & 100 & 100 & 100 & \\
\hline
\end{tabular}

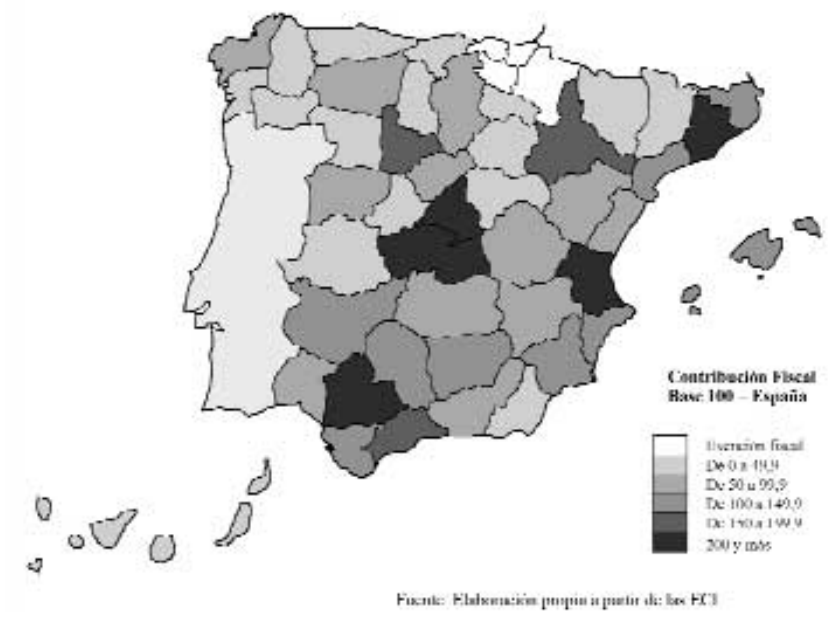

Figura 1. Distribución provincial de las industrias de cerámica y vidrio en España según su contribución fiscal, 1856.

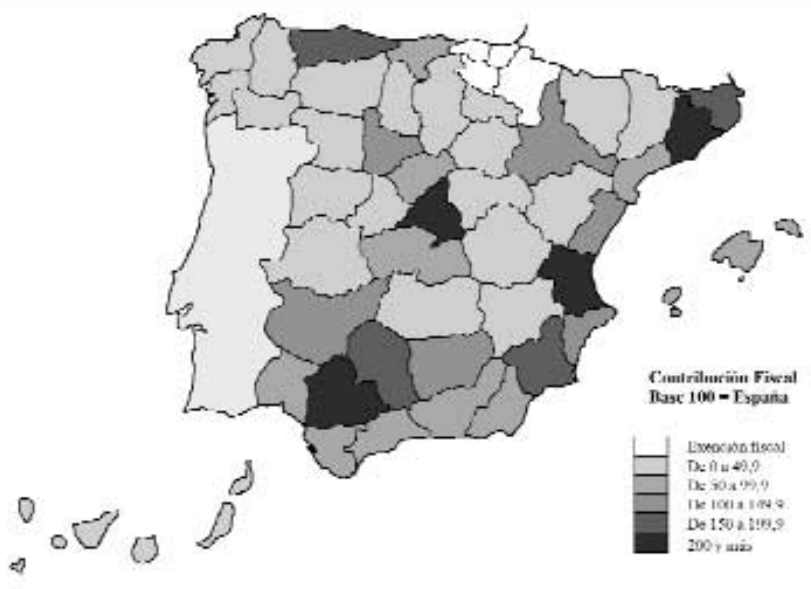

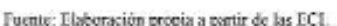

Figura 2. Distribución provincial de las industrias de cerámica y vidrio en España según su contribución fiscal, 1895.

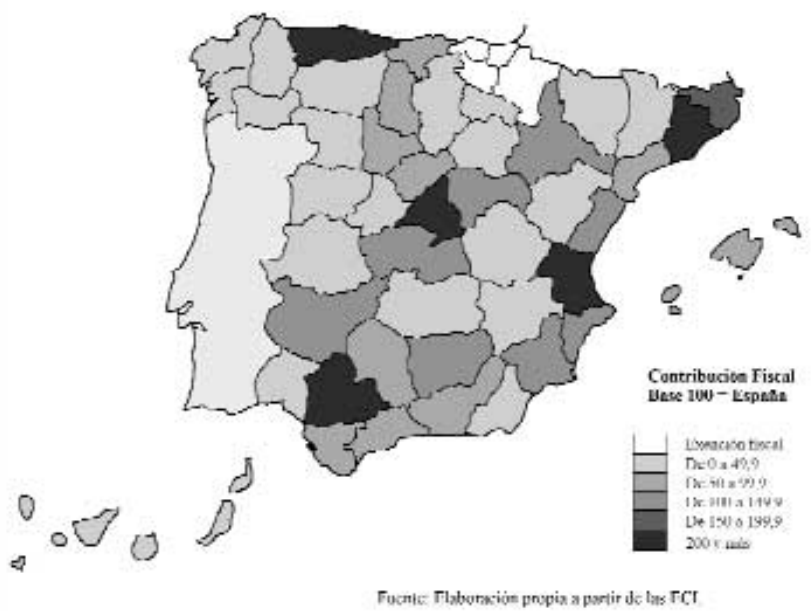


1890 y abandona su posición de líder a comienzos del siglo XX. Andalucía pierde la hegemonía, pero logra mantener una cuota importante. Entre las que más pierden se encuentran las dos Castillas, Aragón y Galicia. La trayectoria del sector en estas regiones corría paralelamente al de su desarrollo económico e industrialización en general. Durante el Antiguo Régimen, sobre todo en el siglo XVIII, habían gozado de ventajas comparativas y mantenido parte de su tejido industrial, incluso hasta mediados del siglo XIX. A partir de este periodo entran en un proceso de deterioro de su renta per capita y de los niveles de bienestar parejo al proceso de desindustrialización ${ }^{9}$. El ferrocarril y los prósperos mercados de oferta de las industrias del litoral mediterráneo fueron desplazándolas de los polos de crecimiento industrial que el capitalismo favoreció en las periferias desde 1860. Cataluña se convirtió, entonces, en la "fábrica de España", descollando también en el sector analizado, y le siguieron el País Vasco, Asturias y las regiones del levante mediterráneo. Más certeramente, fueron las provincias de Oviedo, Barcelona, Valencia y Murcia las que mayor empuje industrial cobraron al final del periodo. Andalucía, incluso, se descolgó habiendo atravesado una anticipada industrialización y entró en una dinámica de subdesarrollo que se acentuó a lo largo de la etapa contemporánea. Sólo Sevilla mantuvo una posición de líder entre 1856 y 1905 (Figuras 2 y 3).

Los factores que explican la localización geográfica y la formación de distritos industriales especializados fueron muy diversos. La localización de las industrias se explica en buena por la cercanía a las fuentes de materias primas y energías. La disponibilidad de tierras arcillosas supone, sin duda, ventajas comparativas en la producción de cerámicas, y los yacimientos de arenas, feldespatos, dolomías y calizas, así como la planta de producción de carbonato sódico lo son para el vidrio. Sin embargo, el acceso al carbón y la hulla de coque para los hornos, la existencia de canales de comercialización y puertos para su exportación, y las ventajas que proporciona la existencia de mano de obra artesanal o cualificada, capitales y tecnologías que, por lo general, se encontraban en las zonas industriales y mineras, constituyen elementos que tener en cuenta. La disponibilidad de energía y el acceso a combustibles baratos y asequibles de acuerdo con la tecnología al uso ha condicionado el emplazamiento de las industrias del sector ${ }^{10}$. A los factores de oferta se sumaron los factores de demanda, entre los que destacan la expansión de los centros urbanos y la construcción de edificios, el aumento de la renta y los gustos de las clases sociales emergentes, fenómenos que tuvieron una mayor dimensión en el curso del siglo XIX. El peso de la demanda que ejercen los cinturones industriales y urbanos se deja sentir en la localización de las industrias del sector.

Como ocurrió con otros sectores industriales emergentes, las regiones mediterráneas y cantábricas presentaron ventajas comparativas derivadas de sus rentas de situación y de la cercanía a las fuentes de suministro de materias primas y combustible. En algunos casos, los proyectos cerámicos y vidrieros que se llevan a cabo dentro del territorio hispano suponen una clara voluntad de eliminar, o erosionar al menos, la fuerte dependencia del sector respecto de las importaciones extranjeras, sobre todo de las lozas inglesas y de los vidrios franceses y belgas ${ }^{11} \mathrm{y}$ la posibilidad de realizar negocios en el mercado interior por parte de los empresarios españoles. El proteccionismo reinante en las décadas centrales y de finales del siglo XIX pudo favorecer esas tentativas. Como se ha visto luego, algunos de los más importantes se realizaron por iniciativa de capitales y compañías extranjeras (Pickman en Sevilla, por la rama de las lozas, y la Compagnie Générale des Verreries Espagnoles en Bilbao por la rama de vidrio plano, por citar dos ejemplos).
TAbla 5. Composición del SeCtor de la Cerámica, VidRio y CAL eN ESPAÑA, 1856-1905. (PORCENTAJES SEGÚN LA CUOTA DE CONTRIBUCIÓN INDUSTRIAL DENTRO DEL SECTOR).

\begin{tabular}{|l|c|c|c|c|}
\hline FABRICAS de & $\mathbf{1 8 5 6}$ & $\mathbf{1 8 9 0}$ & $\mathbf{1 9 0 0}$ & $\mathbf{1 9 0 5}$ \\
\hline Porcelana y loza & 5.6 & 7.6 & 8.8 & 8.9 \\
\hline Tinajas y vasijas & 23.5 & 16.8 & 12.6 & 8.7 \\
\hline Objetos cerámicos & -- & 0.5 & 0.8 & 1.2 \\
\hline Objetos refractarios & -- & 0.3 & 1.1 & 1.3 \\
\hline Azulejos y losetas finas & 1.7 & 3.6 & 4.4 & 5.3 \\
\hline Tejas, ladrillos & 40.7 & 39.8 & 32.6 & 29.9 \\
\hline Losetas hidráulicas & -- & -- & 4.4 & 8.8 \\
\hline Piedra artificial & 0.5 & 0.5 & 1.8 & 4.3 \\
\hline Yeso o cal & 21.9 & 24.1 & 22.7 & 18.3 \\
\hline Cristal & 2.1 & 3.0 & 6.3 & 7.6 \\
\hline Vidrio & 3.9 & 3.7 & 4.3 & 5.5 \\
\hline Total Sector & 100.0 & 100.0 & 100.0 & 100.0 \\
\hline
\end{tabular}

Fuente: $E C I$, elaboración propia. Los porcentajes son datos relativos a la cuota de cada una de las ramas fabriles por concepto de "fabricación" dentro del conjunto del sector de la cerámica, vidrio, cal y yeso. La cuantía de las cuotas se fijaba de acuerdo a los aparatos instalados y que estaban en funcionamiento, ya fueran manuales o mecánicos.

\section{LA COMPOSICIÓN INTERNA DEL SECTOR EN ESPAÑA}

La mayor parte de las industrias del sector en España durante la segunda mitad del siglo XIX responden a la demanda de materiales para la construcción de edificios. Por tanto, son las fábricas de tejas, baldosas y ladrillos, así como las de yeso y cal las que mantienen el impulso del sector hasta comienzos de la década de 1890 (Tabla 5). Hasta ese año, el peso de éstas supone dos tercios en concepto de cuota por fabricación. Es más, la moderna construcción ha impulsado el avance de las fábricas de yeso y cal hasta finales del siglo XIX. Las fábricas de cerámica y vidrio han tenido un peso mucho menor pero, en las dos últimas décadas del siglo, van desplazando a los sectores más tradicionales, como la vasijas y tinajería.

En efecto, hacia 1900, aumentan las fábricas de losetas hidráulicas, piedra artificial y pavimentación, azulejos, mosaicos y losetas finas. Se impone la reconversión de las antiguas manufacturas de mampostería y revestimiento. Los antiguos oficios de caleros ceden ante yeseros y canteros. Las fábricas de piedra artificial cobran un fuerte crecimiento en la primera década del siglo XX. El ritmo de la urbanización impone, no obstante, el recurso a los procedimientos más tradicionales hasta finales del siglo XIX, como advierten los datos. Desde Gerona a Badajoz proliferan pequeñas fábricas de cerámica asentadas en tierras arcillosas, y se amplía el número de las dedicadas a la fabricación de tejas, ladrillos y baldosas. Cataluña y el País Valenciano juegan un papel destacado en la transformación industrial de estas producciones.

La azulejería y la cristalería, ramas de la moderna industria de materiales de construcción para viviendas, adquieren las mayores tasas de crecimiento entre 1856 y 1905 . Empuje que vino dado, en ambos casos, por la expansión de una demanda al calor de la mejora de las rentas, de la urbanización, de las nuevas formas y estilos en la concepción de los hogares, de la fabricación de las casas y edificios. El interés por la higiene y la salubridad pública que recorrió Europa desde 1860, y que propagó la corriente denominada "hygiéniste", formada básicamente por médicos y reformadores preocupados por la cuestión social, concebía grandes avenidas, construcciones mejor 
compartimentadas, espacios abiertos y, sobre todo, más y grandes ventanales. Las modernas fabricas de cristales para ventanas, espejos y la azulejería fueron, sin duda, algunos de los negocios emergentes de fin de siglo.

Igualmente, los cambios promovidos en la oferta tecnológica supusieron economías de escala y precios más competitivos. La producción mecanizada invadió paulatinamente las empresas de cerámica y vidrio en el último tercio del siglo XIX, y descolló particularmente entre la azulejería. En esta rama, la sustitución de artefactos artesanales y manuales por instrumentos mecánicos se fortalece a partir de la década de 1890, destaca "la optimización del proceso de conformación por vía plástica (extrusionadora) y por vía semiseca (con la prensa para polvo humedecido), recuperando la decoración a trepa y aumentando la capacidad de los hornos en la cocción intermitente, y a comienzos del siglo XX, la cocción continúa"12 La introducción de prensas mecánicas, inducidas por vapor, data de 1855 y se registra en el complejo Pickman de la Cartuja (Sevilla). En décadas posteriores, la influencia alcanza a la azulejería valenciana. La Exposición Universal de Barcelona de 1888 fue, probablemente, un auténtico escaparate de los avances técnicos de la época y verdadero acicate de la modernización del sector ${ }^{13}$. Antes, algunas empresas emblemáticas de la azulejería habían llamado la atención del público y recibido premios internacionales ${ }^{14}$. Estas exposiciones, fueran nacionales o internacionales, sirvieron para propiciar, a medio plazo, la renovación tecnológica de la azulejería, el vidrio y el cristal, siendo los sectores más avanzados del sector en las dos últimas décadas del siglo XIX. En cualquier caso, la aparición de nuevas fuentes de energía o de sistemas de aprovechamiento energético cooperó a ello. La industria gasista instalada en España, a partir de 1860, los motores Diesel y la difusión de la electricidad, posteriormente, disputaron a los pesados motores de vapor la alimentación de energía de numerosos talleres, pequeñas empresas y firmas de prestigio.

La azulejería española se había concentrado, a mediados del siglo XIX, en Valencia y Barcelona, pero, a finales del siglo, fueron las provincias de Castellón y Sevilla las que tuvieron mayor expansión (Tabla 6). Entre 1900 y 1905, Valencia pierde la hegemonía que había presenciado durante mucho tiempo y Barcelona, aunque mantuvo cierto empuje fabril hasta la década de 1880, cede sus posiciones relativas en la última década del siglo XIX a favor de Sevilla. Definitivamente, Castellón se impone como la principal zona de la azulejería española. A la luz de los datos, puede señalarse que Castellón y Valencia fueron las áreas de mayor especialización industrial y se convirtieron en los centros productores más importantes del país durante el último tercio del siglo XIX. En la región de Murcia la azulejería es testimonial, documentándose sólo una fábrica a mediados de la década de 1890.

Los municipios de Onda, en Castellón, y algunas localidades de L'Horta de Valencia, como Manises, Alcudia de Crespins, Burjassot son los focos más dinámicos. Con el tiempo, Onda se convierte en el centro más representativo de la azulejería valenciana, que es como decir española. Los orígenes se remontan a las décadas de 1840, probablemente, pero la fuerte y temprana especialización de la zona en industrias de loza fina y cerámica podría explicar su posterior especialización en fábricas de azulejos. Un precedente ilustrado fue, sin duda, la creación de la Real Fábrica de Loza del conde de Aranda en la villa de Alcora en 1727. La dotación de materias primas -zona rica en arcilla- y la existencia de una tradición alfarera -mano de obra cualificada, técnicos y, asimismo, capitales- crearon una atmósfera o 'cultura' industrial favorable al desarrollo fabril de la
TABLA 6. DisTRIBUCIÓN TERRITORIAL DE LA INDUSTRIA DE AZULEJOS EN ESPAÑA, 1856-1905. COMPOSICIÓN RELATIVA (\%) SEGÚN LA CONTRIBUCIÓN FISCAL DE LAS EMPRESAS.

\begin{tabular}{|l|c|c|c|c|c|}
\hline & $\mathbf{1 8 5 6}$ & $\mathbf{1 8 7 9}$ & $\mathbf{1 8 9 5}$ & $\mathbf{1 9 0 0}$ & $\mathbf{1 9 0 5}$ \\
\hline Barcelona & 15,79 & 19,35 & 3,22 & 3,86 & 7,47 \\
\hline Castellón & 10,52 & 29,03 & 38,61 & 38,61 & 46,96 \\
\hline Ciudad-Real & -- & -- & -- & 3,09 & -- \\
\hline Guadalajara & -- & -- & 1,61 & -- & -- \\
\hline Madrid & -- & -- & 4,83 & -- & -- \\
\hline Murcia & -- & -- & 1,61 & -- & -- \\
\hline Segovia & -- & -- & -- & -- & 1,49 \\
\hline Sevilla & 5,27 & 3,23 & 6,43 & 9,27 & 14,94 \\
\hline Teruel & -- & -- & -- & -- & 1,49 \\
\hline Toledo & 5,27 & 3,23 & -- & -- & -- \\
\hline Valencia & 63,16 & 45,16 & 43,70 & 40,15 & 19,42 \\
\hline Zaragoza & -- & -- & -- & 1,54 & 2,24 \\
\hline ESPAÑA & 100,00 & 100,00 & 100.00 & 100,00 & 100,00 \\
\hline
\end{tabular}

Fuente: Elaboración propia a partir de las ECI

cerámica en la provincia de Castellón, que benefició, con el tiempo, la implantación de distritos industriales. Las innovaciones tecnológicas se difundieron a finales del siglo XIX. Algunas fábricas incorporan el vapor y los nuevos sistemas de elaboración. El avance de la rama en el conjunto del sector (Tabla 5) a comienzos del siglo XX hace que la azulejería se constituya en "uno de los sectores más capitalizados de la industria valenciana"15. En general, el País Valenciano se conforma como región especializada en cerámica que registra, asimismo, un proceso de fuerte actividad inventiva como refleja la evolución de las patentes registradas en el sector a lo largo de la centuria ${ }^{16}$.

En la región de Murcia, las industrias de materiales de construcción y alfarería, a pesar de su peso fiscal, apenas merecen mención dado su carácter artesanal y tradicional. La importancia histórica del sector estuvo ligada, sin duda, a la formación de un complejo vidriero-cerámico en el término de Cartagena, que creció al amparo de la industrialización de la cuenca minera entre las décadas de 1840 y 1890. La relevancia que adquirieron las "artes industriales", en concreto el cristal y las lozas cartageneras, fue puesta de manifiesto hace tiempo por los historiadores del arte ${ }^{17}$. Vinculados a la familia Valarino, despuntaron dos establecimientos fabriles construidos en fechas tempranas, la "Fábrica de Cristal y Vidrio de Santa Lucía" (1834-1955) y "La Amistad" (1842-1893), dedicada ésta a la producción de loza. Ambas, junto a "La Cartagenera Industrial Cerámica", de vida efímera (1880-1883), constituyeron la avanzadilla del sector en el sureste español. La primera de ellas fue pionera en el siglo XIX y alcanzó, por su capacidad tecnológica y producción, el primer puesto en el ranking de las industrias vítreas instaladas en el territorio español a comienzos del siglo XX. En la actualidad, apenas quedan vestigios de su pasado y la especialización industrial pasa por las industrias agroalimentarias.

\section{CONCLUSIONES.}

Se ha resaltado la contribución de las industrias englobadas en el sector denominado como 'cerámica, vidrio y materiales de construcción' dentro de la industria fabril española y de la región de Murcia, en particular, durante la segunda mitad del siglo XIX. El examen de los datos, ponderados por ramas 
y sectores y en el contexto de las regiones, pone de manifiesto su temprana especialización productiva. El sector tuvo mayor protagonismo en las regiones periféricas $\mathrm{y}$, al final del periodo, en las mediterráneas, debido a que gozaban de rentas de situación y de ventajas comparativas derivadas de los favorables factores de oferta y demanda. Entre los primeros, fueron determinantes, el acceso a los combustibles, la dotación de materias primas de calidad y la existencia de vías de transporte y comercialización. Entre los segundos, debió mediar una creciente red urbana e industrial que requería de materiales diversos de construcción, ahora más modernos, y unos grupos sociales con mayor renta y de estilos de vida que demandaban todo tipo de artículos para la construcción y embellecimiento de las viviendas, de ornamentación y decoración para el hogar, las necesidades del ajuar y el consumo doméstico. En los albores de la moderna industrialización, mientras entraban en declive, al menos en términos relativos, las fábricas de materiales más tradicionales de la construcción, como yeso y cal, tejas y ladrillos, tinajas y vasijas de barro, cobraron empuje los sectores nuevos, asociados al incremento de la urbanización y mejora de la renta, como las fábricas de cristal y vidrio, de porcelana y loza, de losetas hidráulicas y azulejos, de objetos cerámicos refractarios y de piedra artificial. La mayor parte de estos nuevos sectores estaban ligados a la mayor capitalización, la difusión de máquinas y nuevas tecnologías, y asimismo precisadas de cambios en las formas de organización del trabajo y gestión empresarial.

\section{AGRADECIMIENTOS}

Este texto se ha beneficiado de los comentarios de Alicia Durán, Emilio Criado y de los asistentes al XL Congreso de la Sociedad Española de la Cerámica y el Vidrio a una comunicación presentada en el mismo, celebrado en Onda (Castellón), 8-11 de noviembre de 2000. Una versión de este texto fue presentada al Homenaje de Jordi Nadal, Universidad de Barcelona, 1999 y como Documento de Trabajo de la Facultad de Economía y Empresa de la Universidad de Murcia, mayo de 2001.

\section{BIBLIOGRAFÍA}

1. M.J. Aragoneses (1959): "La Amistad (1845-1893 y la problemática de sus motivos cerámicos", Arte Español. Revista de la Sociedad Española de Amigos del Arte, Madrid, año XLII, XVII de la tercera época, tomo XXII, primer cuatrimestre, pp. 129-143.

2. M.J. Aragoneses (1960a): Artes industriales cartageneras. Lozas del siglo XIX. Museo Arqueológico de Cartagena, Cartagena.

3. M.J. Aragoneses (1960b): "Lozas Españolas: La Cartagenera (1880-1883)", Archivo Español de Arte, t. XXXIII no 129, enero-marzo, pp. 45-54.

4. M.J. Aragoneses (1961): "Pavimentos decimonónicos de azulejería valenciana en Murcia y su provincia", Murgetana, XVII, pp. 29-56.

5. E. Calandre (1949): "La loza de Cartagena", Archivo Español de Arte, t. XXII, nº 87, Madrid, pp. 239-252

6. E. Cañavate Navarro(1958): "Vidrios cartageneros del siglo XIX", Murgetana, XI, pp. 6174.

7. A. Carreras (1990), Industrialización española: estudios de historia cuantitativa. Espasa-Calpe, Madrid.

8. V. J. Estall i Poles (2000), Catálogo de la colección de azulejos de serie del siglo XIX. SECV- Faenza Editrice Ibérica, Castellón.

9. V. J. Estall i Poles, J.L. Porcar (2000): “El desarrollo industrial y tecnológico durante el siglo XIX hasta el primer tercio del siglo XX, en Catálogo de la ruta de la Cerámica, Castellón.

10. M. T. Esteban Señís (1966): "La minería cartagenera, 1840-1919. Aspectos económicos y sociales", Hispania, 101, pp. 61-95.

11. L. Germán, E. Llopis, J. Maluquer de Motes, S. Zapata (eds.) (2001): Historia económica de las regiones españolas, siglos XIX y XX. Barcelona, Crítica.

12. N. Lamoreaux, R. Sokoloff, L. Kenneth (2000): "The Geography of Invention in the American Glass Industry, 1870-1925", Journal of Economic History, 60, 3, pp. 700-729.
13. J. M. Martínez Carrión (1992): "Fuentes para el análisis regional de la industrialización española", Estudis Baleàrics, 43, Institut d'Estudis Baleàrics, Baleares, pp. 27-44.

14. J.M. Martínez Carrión (1997): “La industrialización española y las fuentes fiscales. Una valoración regional desde la Contribución Industrial y de Comercio,1856-1950", en Actas del VI Congreso de la Asociación de Historia Económica, Girona.

15. F. A. Martínez Gallego (1995): Desarrollo y crecimiento. La industrialización valenciana, 18341914. Generalitat Valenciana, Conselleria d'Indústria, Valencia.

16. J. Nadal (1972): "Industrialización y desindustrialización del sureste español, 1817-1913", Moneda y Crédito, 120, pp. 3-80 [reeditado en A. Parejo y A. Sánchez (eds.,), Economía andaluza e historia industrial. Estudios en homenaje a Jordi Nadal. Colección Flores de Lemus, Motril, pp. 65-140.

17. J. Nadal (1975): El fracaso de la revolución industrial en España (1814-1913). Ariel, Barcelona.

18. J. Nadal (1987): "La industria fabril española en 1900. Una aproximación", en J. Nadal, A. Carreras, y C. Sudrià (compiladores), La economía española en el siglo XX. Una perspectiva histórica. Ariel, Barcelona, pp. 23-61.

19. J. Nadal (1992): Tejer, moler y fundir. Estudios de historia industrial. Ariel, Barcelona.

20. J. Nadal y A. Carreras (dirección y coordinación) (1990), Pautas de industrialización española (siglos XIX y XX), Ariel, Barcelona.

21. J. Nadal y J. Catalán (eds.,) (1994): La cara oculta de la industrialización española. La modernización de los sectores no líderes. Alianza Editorial, Madrid.

22. J. M. Ortiz-Villajos (1999): “Tecnología y desarrollo regional en España, 1882-1935. Estudio basado e n patentes de invención", Revista de Historia Económica, XVII, 1, pp. 1150.

23. J. Pérez Camps (2000), “La evolución de la azulejería valenciana entre 1860 y 1936”, en Catálogo de la Ruta de la Cerámica, Castellón.

24. M. T. Pérez Picazo (1990), "Pautas de industrialización de la región murciana. Del textil al agroalimentario", en J. Nadal y A. Carreras, coord., Pautas de industrialización española (siglos XIX y XX), Ariel, Barcelona, pp. 315-340.

25. J. P. Sáiz González (1999): "Patentes, cambio técnico e industrialización en la España del siglo XIX", Revista de Historia Económica, XVII, 2, pp. 265-303.

26. J. Sierra Álvarez (1993): El complejo vidriero de Campóo (Cantabria), 1844-1928. Cámara Oficial de Comercio, Industria y Navegación de Cantabri, Santander.

27. G. Tortella Casares (1994), El desarrollo de la España Contemporánea. Historia económica de los siglos XIX y XX. Alianza editorial, Madrid.

28. J. B. Vilar y P. Egea Bruno (1989): La minería murciana contemporánea (1840-1930), Universidad de Murcia, Murcia.

\section{NOTAS}

1. J. Nadal, 1975.

2. A partir de 1905 , las ECI comienzan a ser menos fiables, dado que las empresas formadas por sociedades anónimas tributan por concepto diferente. Aunque la mayor parte de las empresas son de tipo familiar en las primeras décadas del siglo XX, no se puede desdeñar la contribución de las sociedades anónimas a la formación de capital industrial en el sector de la cerámica y el vidrio. Por tanto, cualquier análisis histórico realizado con estas fuentes para el primer tercio del siglo XX debe ponderarse con otras estadísticas y fuentes.

3. La bondad de estas estadísticas fiscales ha sido señalada por J. Nadal en diversos estudios sobre la industrialización española, J. Nadal, 1987, pp. 23-61. Una crítica de las Estadísticas de Contribución Industrial puede verse en Martínez Carrión, 1992, pp. 27 44; y Martínez Carrión, 1997.

4. Nadal y Carreras, 1990; Nadal y Catalán, 1994.

5. Los estudios de historia económica regional han destacado el peso de las industrias alimentarias y textiles, así como de la metalúrgicas, en la línea de la tradición historiográfica española, y apenas han señalado indicios de su desarrollo en Murcia, ni la contribución del sector que analizamos, véase Pérez Picazo, 1990, pp. 314-341.

6. Entre los estudios, destacan Esteban Senís, 1966; Nadal, 1972; Vilar y Egea, 1989; Guillén Riquelme, 1997.

7. Nadal, 1987.

8. La cuota aumentó en 1895 a 292.038 pesetas siendo por estos años los de mayor recaudación del siglo XIX.

9. Carreras, 1990; Germán, Llopis, Maluquer de Motes y Zapata, (eds.), 2001.

10. En Estados Unidos, el emplazamiento de la manufactura del vidrio estuvo ligado a la localización de fuentes importantes de carbón vegetal hasta que se desarrolló la industria del gas natural a mediados del siglo XIX. La nueva situación provocó cambios significativos y al emigración de las industrias hacia los territorios de la nueva fuente energética. Ello explica, por ejemplo, el fuerte desarrollo de la industria vidriera en Ohio y el Medio Oeste en las décadas de 1870-1880, Lamoreaux y Sokoloff, 2000, p. 703-705.

11. Nadal, 1992, p. 290.

12. Estall i Poles, 2000, p. 20

13. La Exposición de Barcelona reunió a 12.233 expositores, siendo de las menos significativas de las que realizaron en el siglo XIX (la primera fue en Londres, 1851) y fue visitada por 1.227.000 de personas.

14. Estall i Poles, 2000; y Pérez Camps, 2000.

15. Martínez Gallego, 1995, pp. 167-186.

16. Sobre la relación entre el desarrollo industrial y las innovaciones tecnológicas a través de las patentes de invención entre 1800 y 1935, véase Sáiz González (1999) y Ortiz-Villajos (1999).

17. Sobre vidrios cartageneros, véase el trabajo de Cañabate Navarro, 1958; sobre la industria de lozas en Cartagena, véase Calandre, 1949; y Aragoneses, 1959, 1960a y 1960b. 
APÉNDICE I: CONTRIBUYENTES Y CUOTAS POR FABRICACIÓN EN EL SECTOR DE ‘CERÁMICA, VIDRIO Y MATERIALES DE CONSTRUCCIÓN’, 1856.

$\begin{array}{lrrrr} & \begin{array}{c}\text { Contribuyentes } \\ \text { (número) }\end{array} & \begin{array}{c}\text { Cuotas } \\ \text { (en reales) }\end{array} & \begin{array}{c}\text { Contribuyentes } \\ \%\end{array} & \begin{array}{c}\text { Cuotas } \\ \%\end{array} \\ \text { Albacete } & & & & \\ \text { Alicante } & 76 & 7.101 & 3,9 & 1,4 \\ \text { Almería } & 154 & 14.047 & 7,8 & 2,7 \\ \text { Avila } & 36 & 3.566 & 1,8 & 0,7 \\ \text { Badajoz } & 42 & 4.107 & 2,1 & 0,8 \\ \text { Barcelona } & 163 & 14.546 & 8,3 & 2,8 \\ \text { Burgos } & 423 & 45.860 & 21,5 & 8,8 \\ \text { Cáceres } & 45 & 5.962 & 2,3 & 1,2 \\ \text { Cádiz } & 62 & 5.143 & 3,1 & 1,0 \\ \text { Castellón } & 151 & 13.607 & 7,7 & 2,6 \\ \text { Ciudad-Real } & 79 & 9.119 & 4,0 & 1,8 \\ \text { Córdoba } & 117 & 7.938 & 5,9 & 1,5 \\ \text { Coruña } & 183 & 16.399 & 9,3 & 3,2 \\ \text { Cuenca } & 38 & 7.578 & 1,9 & 1,5 \\ \text { Gerona } & 70 & 6.174 & 3,6 & 1,2 \\ \text { Granada } & 206 & 17.224 & 10,5 & 3,3 \\ \text { Guadalajara } & 79 & 7.672 & 4,0 & 1,5 \\ \text { Huelva } & 58 & 4.177 & 2,9 & 0,8 \\ \text { Huesca } & 83 & 5.936 & 4,2 & 1,1 \\ \text { Jaén } & 37 & 4.480 & 1,9 & 0,9 \\ \text { León } & 146 & 12.842 & 7,4 & 2,5 \\ \text { Lérida } & 105 & 8.764 & 5,3 & 1,7 \\ \text { Logroño } & 55 & 5.171 & 2,8 & 1,0 \\ \text { Lugo } & 24 & 2.679 & 1,2 & 0,5 \\ \text { Madrid } & 27 & 5.185 & 1,4 & 1,0 \\ \text { Málaga } & 221 & 45.784 & 11,2 & 8,8 \\ \text { Murcia } & 188 & 20.912 & 9,5 & 4,0 \\ \text { Orense } & 112 & 12.636 & 5,7 & 2,4 \\ \text { Oviedo } & 10 & 984 & 0,5 & 0,2 \\ \text { Palencia } & 3 & 2.861 & 0,2 & 0,6 \\ \text { Pontevedra } & 59 & 4.266 & 3,0 & 0,8 \\ \text { Salamanca } & 23 & 1.727 & 1,2 & 0,3 \\ \text { Santander } & 89 & 7.443 & 4,5 & 1,4 \\ \text { Segovia } & 25 & 3.105 & 1,3 & 0,6 \\ \text { Sevilla } & 65 & 7.901 & 3,3 & 1,5 \\ \text { Soria } & 333 & 46.439 & 16,9 & 9,0 \\ \text { Tarragona } & 14 & 1.727 & 0,7 & 0,3 \\ \text { Teruel } & 135 & 13.165 & 6,9 & 2,5 \\ \text { Toledo } & 36 & 2.959 & 1,8 & 0,6 \\ \text { Valencia } & 372 & 28.027 & 18,9 & 5,4 \\ \text { Valladolid } & 263 & 27.586 & 13,4 & 5,3 \\ \text { Zamora } & 210 & 18.720 & 10,7 & 3,6 \\ \text { Zaragoza } & 70 & 5.614 & 3,6 & 1,1 \\ \text { Islas Baleares } & 180 & 18.578 & 9,1 & 3,6 \\ \text { Canarias } & 90 & 11.649 & 4,6 & 2,2 \\ \text { ESPAÑ A } & 4.970 & 518.279 & 100,0 & 100,0 \\ & & 919 & & \end{array}$

APÉNDICE II: CONTRIBUYENTES Y CUOTAS POR FABRICACIÓN EN EL SECTOR DE ‘CERÁMICA, VIDRIO Y MATERIALES DE CONSTRUCCIÓN’, 1895.

Fuente: ECI

\begin{tabular}{|c|c|c|c|c|}
\hline & $\begin{array}{l}\text { Contribuyentes } \\
\text { (número) }\end{array}$ & $\begin{array}{c}\text { Cuota } \\
\text { (en pesetas) }\end{array}$ & $\begin{array}{c}\text { Contribuyentes } \\
\%\end{array}$ & $\begin{array}{c}\text { Cuota } \\
\%\end{array}$ \\
\hline Albacete & 72 & 2.184 & 1,27 & 1,14 \\
\hline Alicante & 142 & 8.171 & 2,51 & 4,25 \\
\hline Almería & 68 & 4.447 & 1,20 & 2,32 \\
\hline Avila & 67 & 1.765 & 1,18 & 0,92 \\
\hline Badajoz & 226 & 7.871 & 3,99 & 4,10 \\
\hline Barcelona & 558 & 41.932 & 9,85 & 21,84 \\
\hline Burgos & 63 & 2.603 & 1,11 & 1,36 \\
\hline Cáceres & 47 & 1.521 & 0,83 & 0,79 \\
\hline Cádiz & 108 & 6.028 & 1,91 & 3,14 \\
\hline Castellón & 157 & 7.297 & 2,77 & 3,80 \\
\hline Ciudad-Real & 85 & 2.844 & 1,50 & 1,48 \\
\hline Córdoba & 247 & 10.610 & 4,36 & 5,53 \\
\hline Coruña & 41 & 574 & 0,72 & 0,30 \\
\hline Cuenca & 78 & 1.894 & 1,38 & 0,99 \\
\hline Gerona & 260 & 12.493 & 4,59 & 6,51 \\
\hline Granada & 109 & 3.324 & 1,92 & 1,73 \\
\hline Guadalajara & 50 & 1.578 & 0,88 & 0,82 \\
\hline Huelva & 103 & 4.199 & 1,82 & 2,19 \\
\hline Huesca & 72 & 2.626 & 1,27 & 1,37 \\
\hline Jaén & 232 & 8.106 & 4,10 & 4,22 \\
\hline León & 43 & 925 & 0,76 & 0,48 \\
\hline Lérida & 109 & 3.059 & 1,92 & 1,59 \\
\hline Logroño & 47 & 2.430 & 0,83 & 1,27 \\
\hline Lugo & 27 & 1.037 & 0,48 & 0,54 \\
\hline Madrid & 241 & 19.761 & 4,25 & 10,29 \\
\hline Málaga & 138 & 5.481 & 2,44 & 2,85 \\
\hline Murcia & 234 & 12.840 & 4,13 & 6,69 \\
\hline Orense & 15 & 293 & 0,26 & 0,15 \\
\hline Oviedo & 75 & 11.459 & 1,32 & 5,97 \\
\hline Palencia & 57 & 2.925 & 1,01 & 1,52 \\
\hline Pontevedra & 40 & 929 & 0,71 & 0,48 \\
\hline Salamanca & 89 & 2.871 & 1,57 & 1,49 \\
\hline Santander & 53 & 6.442 & 0,94 & 3,35 \\
\hline Segovia & 41 & 5.442 & 0,72 & 2,83 \\
\hline Sevilla & 316 & 21.991 & 5,58 & 11,45 \\
\hline Soria & 37 & 1.762 & 0,65 & 0,92 \\
\hline Tarragona & 138 & 5.669 & 2,44 & 2,95 \\
\hline Teruel & 37 & 1.300 & 0,65 & 0,68 \\
\hline Toledo & 191 & 5.665 & 3,37 & 2,95 \\
\hline Valencia & 424 & 24.549 & 7,49 & 12,78 \\
\hline Valladolid & 142 & 6.917 & 2,51 & 3,60 \\
\hline Zamora & 95 & 2.473 & 1,68 & 1,29 \\
\hline Zaragoza & 130 & 7.254 & 2,30 & 3,78 \\
\hline Islas Baleares & 132 & 5.257 & 2,33 & 2,74 \\
\hline Canarias & 28 & 1.239 & 0,49 & 0,64 \\
\hline ESPAÑA & 5.664 & 292.038 & 100,00 & 100,00 \\
\hline
\end{tabular}

APÉNDICE III: CONTRIBUYENTES Y CUOTAS POR FABRICACIÓN EN EL SECTOR DE ‘CERÁMICA, VIDRIO Y MATERIALES DE CONSTRUCCIÓN’, 1905.

\begin{tabular}{|c|c|c|c|c|c|c|c|c|c|}
\hline & $\begin{array}{l}\text { Contribuyentes } \\
\text { (número) }\end{array}$ & $\begin{array}{c}\text { Cuota } \\
\text { (en pesetas) }\end{array}$ & $\begin{array}{c}\text { Contribuyentes } \\
\%\end{array}$ & $\begin{array}{c}\text { Cuota } \\
\%\end{array}$ & & $\begin{array}{l}\text { Contribuyentes } \\
\text { (número) }\end{array}$ & $\begin{array}{c}\text { Cuota } \\
\text { (en pesetas) }\end{array}$ & $\begin{array}{c}\text { Contribuyentes } \\
\%\end{array}$ & $\begin{array}{c}\text { Cuota } \\
\%\end{array}$ \\
\hline Albacete & 70 & 2.314 & 1,40 & 0,71 & Lugo & 12 & 534 & 0,24 & 0,16 \\
\hline Alicante & 125 & 7.389 & 2,51 & 2,28 & Madrid & 259 & 27.744 & 5,19 & 8,55 \\
\hline Almería & 56 & 2.665 & 1,12 & 0,82 & Málaga & 87 & 4.417 & 1,74 & 1,36 \\
\hline Avila & 56 & 1.056 & 1,12 & 0,33 & Murcia & 93 & 8.110 & 1,86 & 2,50 \\
\hline Badajoz & 198 & 7.520 & 3,97 & 2,32 & Orense & 18 & 427 & 0,36 & 0,13 \\
\hline Barcelona & 514 & 64.017 & 10,30 & 19,72 & Oviedo & 84 & 19.283 & 1,68 & 5,94 \\
\hline Burgos & 66 & 2.195 & 1,32 & 0,68 & Palencia & 50 & 4.744 & 1,00 & 1,46 \\
\hline Cáceres & 45 & 1.027 & 0,90 & 0,32 & Pontevedra & 36 & 1.090 & 0,72 & 0,34 \\
\hline Cádiz & 71 & 5.646 & 1,42 & 1,74 & Salamanca & 114 & 1.737 & 2,29 & 0,54 \\
\hline Castellón & 162 & 9.474 & 3,25 & 2,92 & Santander & 50 & 5.758 & 1,00 & 1,77 \\
\hline Ciudad-Real & 52 & 1.350 & 1,04 & 0,42 & Segovia & 59 & 3.806 & 1,18 & 1,17 \\
\hline Córdoba & 161 & 5.722 & 3,23 & 1,76 & Sevilla & 317 & 26.308 & 6,36 & 8,10 \\
\hline Coruña & 43 & 626 & 0,86 & 0,19 & Soria & 43 & 1.921 & 0,86 & 0,59 \\
\hline Cuenca & 66 & 1.681 & 1,32 & 0,52 & Tarragona & 98 & 3.797 & 1,96 & 1,17 \\
\hline Gerona & 196 & 12.249 & 3,93 & 3,77 & Teruel & 36 & 1.325 & 0,72 & 0,41 \\
\hline Granada & 94 & 4.095 & 1,88 & 1,26 & Toledo & 247 & 7.328 & 4,95 & 2,26 \\
\hline Guadalajara & 40 & 7.900 & 0,80 & 2,43 & Valencia & 372 & 26.187 & 7,46 & 8,07 \\
\hline Huelva & 72 & 3.401 & 1,44 & 1,05 & Valladolid & 120 & 5.080 & 2,41 & 1,56 \\
\hline Huesca & 57 & 1.848 & 1,14 & 0,57 & Zamora & 69 & 2.135 & 1,38 & 0,66 \\
\hline Jaén & 204 & 7.239 & 4,09 & 2,23 & Zaragoza & 143 & 10.762 & 2,87 & 3,32 \\
\hline León & 46 & 2.496 & 0,92 & 0,77 & Islas Baleares & 110 & 3.947 & 2,21 & 1,22 \\
\hline Lérida & 83 & 2.613 & 1,66 & 0,80 & Canarias & 43 & 1.579 & 0,86 & 0,49 \\
\hline Logroño & 51 & 2.086 & 1,02 & 0,64 & ESPAÑA & 4.988 & 324.628 & 100,00 & 100,00 \\
\hline
\end{tabular}

